\title{
Interaction between seismicity and deformation on different time scales in volcanic areas: Campi Flegrei and Stromboli
}

\author{
Mariarosaria Falanga ${ }^{1}$, Enza De Lauro ${ }^{1}$, Simona Petrosino $^{2}$, and Salvatore De Martino ${ }^{1}$ \\ ${ }^{1}$ Università degli Studi di Salerno, Dipartimento di Ingegneria dell'Informazione ed Elettrica \\ e Matematica applicata/DIEM, Fisciano, Italy \\ ${ }^{2}$ Istituto Nazionale di Geofisica e Vulcanologia, Sezione di Napoli - Osservatorio Vesuviano, Naples, Italy
}

Correspondence: Enza De Lauro (edelauro@unisa.it)

Received: 3 October 2019 - Revised: 22 November 2019 - Accepted: 26 November 2019 - Published: 5 December 2019

\begin{abstract}
We study oscillations recorded at Stromboli and Campi Flegrei by different sensors: seismometers, strainmeters and tiltmeters. We examine both the high-frequency $(>0.5 \mathrm{~Hz}$ ) portion of the spectrum and very long period signals up to tidal scales. In this context, seismicity and deformation are investigated on different time scales (from minutes to days/years) in order to identify the basic elements of their interaction, whose understanding should provide new insights on the predictive models. In this work, the strict relation of tides and volcanic processes is shown.

At Stromboli, indeed the transition from the stationary phase to the non-stationary phase seems to have a tidal precursor that is related to the duration of the crisis. The subsequent volcanic activity is interpreted as the response of the volcano to restore the equilibrium condition. The moveout from equilibrium produces, first, variations in the standard statistics of explosions, then leads to effusive stage and to a pressure drop in the shallow feeding system. That process induces the nucleation of a gas bubble and the excitation of low frequencies. Campi Flegrei seismicity shows a correlation between the diurnal solar solid tide and the energy released by the long period signals, indicating that the whole mechanism is modulated on a tidal scale. In other words, in the case of Stromboli, a departure from the equilibrium state is marked by solid tide variations in a certain frequency band. On the other hand, at Campi Flegrei diurnal to annual solid tides modulate an increase of volcanic activity.
\end{abstract}

\section{Introduction}

A fundamental task in the active volcanic areas is to asses, record, and model the deformations, which the area makes experiences with the ultimate goal to forecast any external or internal source-induced non stationary phenomena. This passes through the analysis of different geophysical parameters which describe a variety of spatial and temporal scales involved. In this work, considerable attention is placed on specific volcanic areas such as Stromboli volcano and Campi Flegrei Caldera. This choice is based on the consideration that these systems are representative of basaltic volcanoes with open conduit (Stromboli) and hydrothermal calderic systems (Campi Flegrei).

Stromboli volcano and Campi Flegrei Caldera belong to the Italy's volcanism. It is an effect of the interaction between the boundary of the Eurasian tectonic plate and the African tectonic plate sits south in the Mediterranean Sea.

Stromboli is a strato-volcano (Francalanci et al., 2000) located in Tyrrhenian sea on the homonymous island at North of the Sicilian coasts (Italy). Its volcanic activity consists of explosion-quakes occurring every 5-10 min that eject gas and scoria superimposed to a continuous persistent volcanic tremor (see, e.g., De Lauro et al., 2008). Stromboli cyclically escapes from this stationary standard behaviour to experience a non-stationary stage. From a seismological point of view, the stationary behaviour is characterized by both a continuous tremor normally distributed in amplitude and by low-intensity explosions with a log-normal amplitude distribution and Poissonian inter-occurrence times (Bottiglieri et al., 2005; De Lauro et al., 2008). During the non-stationary phase, effusive and paroxysmal events and seismic swarms 
may occur, which can likely trigger landslides and tsunami (Gambino and Scaltrito, 2018).

Campi Flegrei $(\mathrm{CF})$ volcanic complex is a nested caldera located in a densely populated area, Southern Italy (Orsi et al., 1996). It was interested by the large Campanian Ignimbrite $(40 \mathrm{kyr} \mathrm{BP} ; \mathrm{VEI}=5)$ and the Neapolitan Yellow Tuff ( 15 kyr BP; VEI $=6$ ) eruptions. CF activity is worldwide known for the phenomenon called "bradyseism", consisting of fast ground uplifts followed by slow subsidence phases. The seismicity consists of Long-period (LP) events, volcano-tectonic (VT) events, hydrothermal tremor, regional tectonic seismicity, anthropogenic and meteo-marine contribution noise and because of the noise due to urban activity, often the volcanic signals are buried (Saccorotti et al., 2007; Capuano et al., 2017).

The role of fluid migration has been recognized as a fundamental mechanism in the shallow dynamics of Campi Flegrei caldera i.e., LP events are originated in a dendritic network of conduits or branches of a hydrothermal system and represent the main self-oscillations; VT are associated with rock fractures (Cusano et al., 2008; Falanga and Petrosino, 2012; De Lauro et al., 2012, 2013; Chiodini et al., 2016).

This work makes use of data from a network of multiparametric continuous sensors including tilmeters, broadband seismometers and strainmeters to study the correlation between deformation and seismicity with the aim of providing a clear picture of:

1. The discrimination between external and internal sources related to the volcano dynamics.

2. The evaluation of the crustal response in terms of ground deformation to external excitations such as long/medium period tidal constituents.

3. The individuation of the synchronization mechanism between the short time scale proper of the seismic activity (explosion quakes and LP events) and longer scales related to the Earth tides.

4. How the low turbulence, i.e. the scales mixing, acts within natural systems. Indeed, these are systems in which the tidal scales interact with the seismic ones.

\section{Deformative and seismic data sets}

Standard and innovative techniques have been applied in frequency and time domain spanning from FFT until methods by using a fourth-order statistic (i.e., Independent Component Analysis-ICA) to a large network of seismic, tiltmeter and dilatometer data acquired by stations deployed both borehole and on surface.

\subsection{Stromboli}

At Stromboli volcano, the data-set consists of the recordings of seismic stations operating in two selected time periods

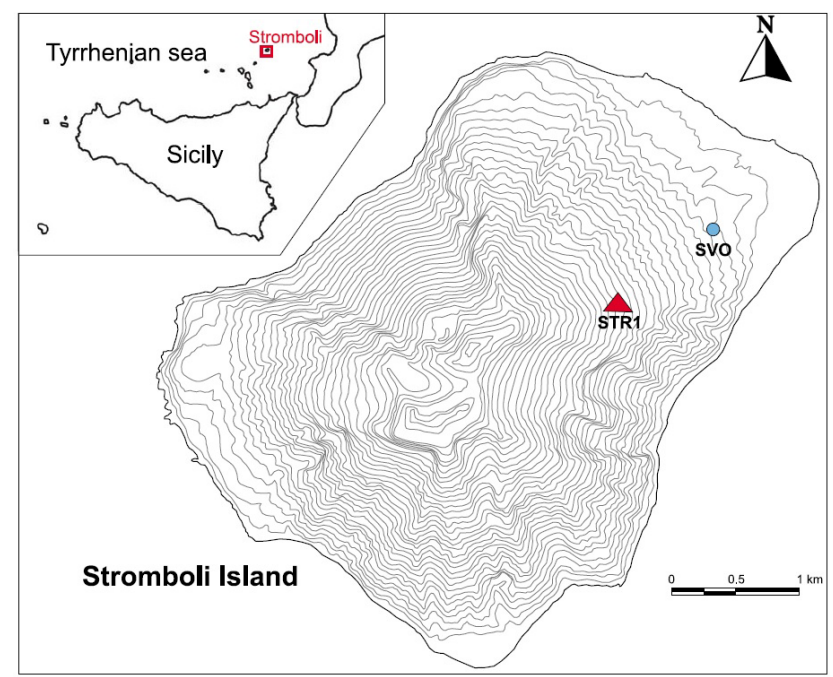

Figure 1. Map of location of both seismometer labelled STR1 and SX15 and strainmeter labelled SVO in 2007.

from 23 May 2002 to 30 January 2003 (SW1) and 7 October 2006-31 March 2007 (SW2). Regarding SW1, data were acquired by a three-component Lennartz 3D-5s seismometer at the station SX15 with a sampling rate of $50 \mathrm{~Hz}$ (De Martino et al., 2011a). The station was located about $2.5 \mathrm{~km}$ far from the craters in NE direction (Fig. 1). The recordings include the first $34 \mathrm{~d}$ of the effusion began on 28 December 2002, and lasted until 6 July 2003 (see e.g., Calvari et al., 2005; Cesca et el., 2007). During the crises, a fissure opened at the base of the NE crater. On 5 April 2003, a paroxysmal explosion occurred in the crater area. The effusion was mainly fed by a fissure at about $550 \mathrm{~m}$ a.s.l. along the Sciara del Fuoco until mid-February 2003, and from a fissure formed $170 \mathrm{~m}$ above since then and until the end of the crisis (De Martino et al., 2012).

With respect to 2007 , the seismic data was acquired by a three-component Guralp CMG-40T broadband seismometer at the station STR1. Moreover, we use data from a strainmeter located close to the local Civil Defense Centre (SVO), spanning the period 20 January-30 June 2007.

\subsection{Campi Flegrei}

The seismic data were collected by the permanent and mobile broadband three-component seismometers of the Campi Flegrei seismic monitoring network, managed by the Istituto Nazionale di Geofisica e Vulcanologia-Osservatorio Vesuviano (INGV-OV) (for details, see Saccorotti et al., 2007). Specifically, the selected LP data were acquired in October 2006 by the three-component Lennartz LE-3D/20s seismometers operating at ASB2, OMN2, and AMS2 sites, and by Guralp CMG40T $60 \mathrm{~s}$ geophones at BGNG and TAGG stations (Fig. 2). The locations of VT earthquakes (Fig. 2) 


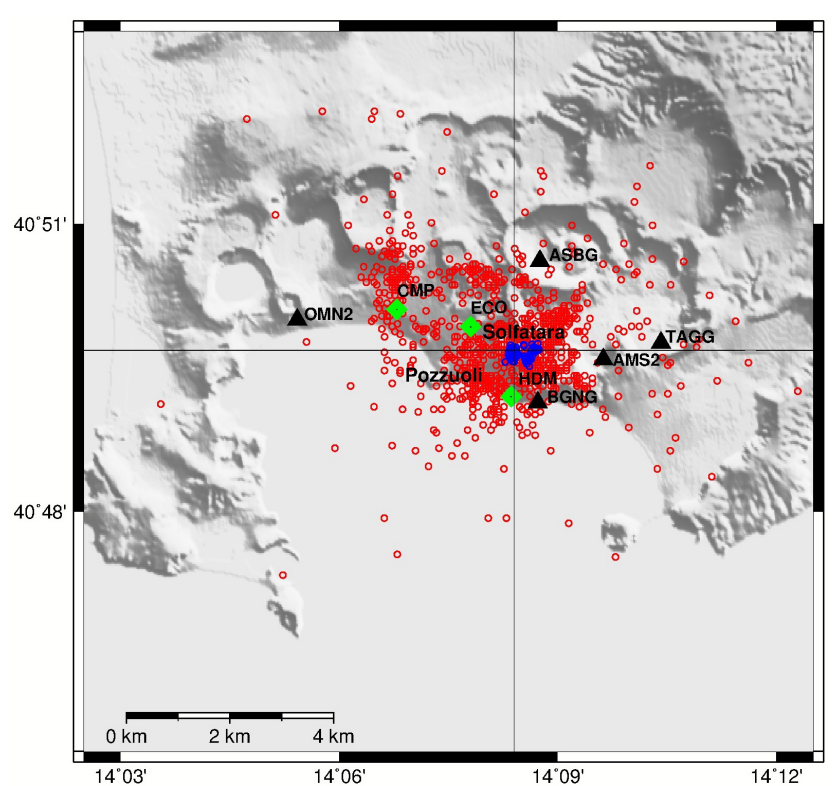

Figure 2. Map of the Campi Flegrei area with the three borehole tiltmeters (green diamonds) and the five seismic stations (black triangles) used for the analysis of the LP seismicity occurred in October 2006. The locations of the LPs in the Solfatara area are shown by blue circles. The map also reports the locations (red circles) of the VT earthquakes recorded by the INGV-Osservatorio Vesuviano seismic network in 2005-2016.

occurred in 2005-2016 were obtained by using data recorded by the whole seismic network (Petrosino et al., 2018).

Ground deformation data were acquired by three borehole digital Lily tiltmeters (HDM, ECO and CMP) installed into $25 \mathrm{~m}$ deep (Fig. 3). They belong to the tiltmeter monitoring network of Campi Flegrei (Ricco et al., 2003; Aquino et al., 2016) consisting of ten stations. The acquisition are made along the EW and NS axes at 1sample/minute. All the stations are distributed around the maximum ground uplift area (corresponding to the city of Pozzuoli) measured during the last three unrest episodes between 1969-1972, 19821984 and 2005-present by the periodical leveling surveys (Del Gaudio et al., 2010).

\section{Deformative and seismic analyses}

\subsection{Stromboli}

Several studies on the seismicity recorded at Stromboli (Bottiglieri et al., 2005; De Lauro et al., 2008), during the stationary phase, showed that the explosion quakes are regulated by the Poissonian processes, which operate on a variety of time scales, whereas the relative amplitudes show a lognormal distribution. The onset of the effusive phase is instead preceded by seismic swarms, whose time distribution is unimodal. The transition mechanism between stationary/nonstationary stages is ruled by the interplay of short and long-

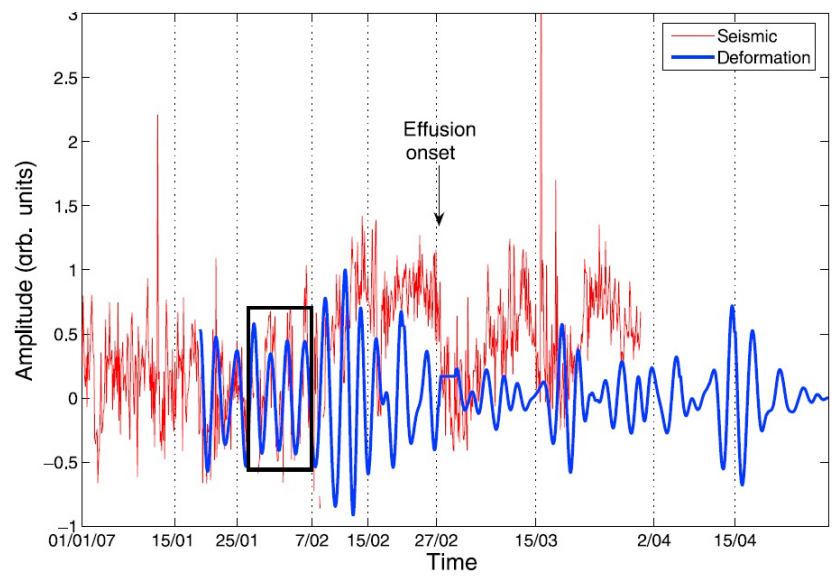

Figure 3. Comparison between the amplitude of the seismic signal (red line) and the deformation (blue line) filtered in the range [24] d. Both the signals were normalized to facilitate the comparison. Dilatometer's recording starts on January 19th. In this representation, the match between the curves evidenced by a black rectangle covers the period 19 January-7 February, when an increase of the explosions' amplitude occurs leading to the effusion (after De Martino et al., 2011b).

period source oscillations. Indeed, De Martino et al. (2011b) relatively to the period 19 January-30 June 2007 identified a $3 \mathrm{~d}$ periodicity both as volumetric deformation signal and as modulation in the explosion's amplitude. The superposition between those two curves becomes strongly correlated in correspondence of the effusion when the explosions' amplitude enhances. This long periodicity plays an important role in the dynamics of the volcanic processes indicating a possible role of the tides in the genesis of the crisis. Before and after this non equilibrium phase, no correlation is present in both seismograms and strains. This result is shown in Fig. 3.

The interest was then to identify which tidal constituent is involved in the process. This is shown in Fig. 4, which shows the time evolution of the spectral peaks of Earth tides, specifically diurnal, semidiurnal and $3 \mathrm{~d}$ constituents as acquired by the strainmeter. As result, the constituents that do not show any variation with respect their mean value over the time are of course non related to the volcanic activity. In our case, diurnal and semidiurnal tides are basically constant; whereas a $3 \mathrm{~d}$ component shows a clear growth at the end of January 2007 and it returns to the stationary value well before the end of effusive phase.

The picture that emerges from the previous results is that of variables of long scale (tides) are sensitive to the arrival of a non-equilibrium phenomenon. In the case of Stromboli, approaching to the non-stationary phase, the statistical parameters (shape of the distribution) of the explosion quakes change, from Poissonian to quasi-periodical process. Moreover, a $3 \mathrm{~d}$ signal appears as a precursor of non-stationary phenomena, due to an increase of the pressure in the system. This is a typical example of scale mixing in natural systems. 


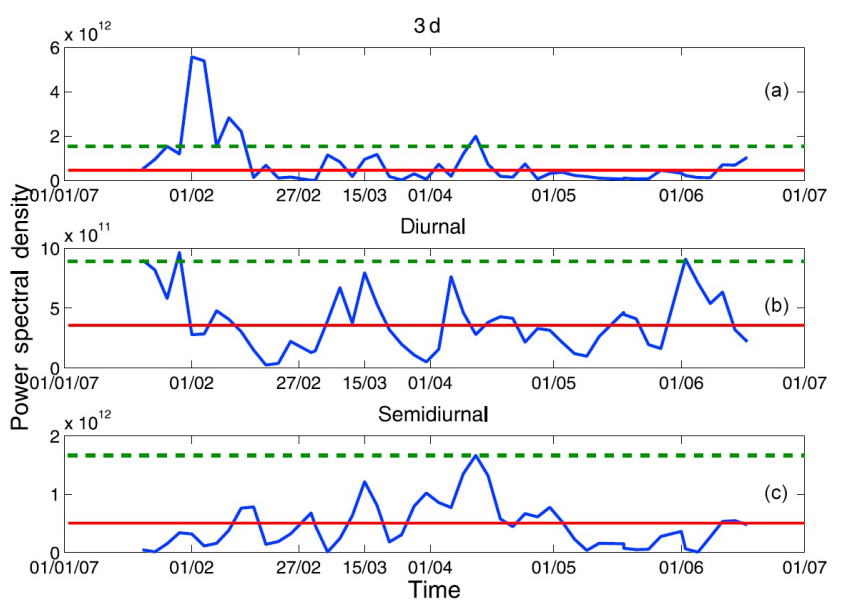

Figure 4. Power Spectral Density (PSD) of the frequencies corresponding to periods of (a) $3 \mathrm{~d}$, (b) $24 \mathrm{~h}$ and (c) $12 \mathrm{~h}$ of the dilatometer in the period January-June 2007 (blue lines). All values fall within the statistical variability except the $3 \mathrm{~d}$ component in the period 25 January-5 February 2007, suggesting a significant deviation of the energy of this frequency only in this period. The red line represents the mean value of the PSD and the green line corresponds to the mean value plus three times the standard deviation (after De Martino et al., 2011b).

These aspects have to be deeply investigated also in other volcanic areas, as e.g. Campi Flegrei, which at least in the shallow part of the caldera is made of a hydrothermal system, and so the system should be more sensitive to a tidal role for the presence of fluids.

\subsection{Campi Flegrei}

The interest of this work is the understanding of the deformation mechanisms also in a different contest, i.e. a hydrothermal area. Useful information to constrain the source mechanism was obtained by observing the release of the seismic energy and how the related elastic vibrations provide some constraints on the charge/discharge processes and on the geometry involved in the solid part. From a seismological point of view, long-term seismic events (LPs) are a direct consequence of the dynamics of the shallow hydrothermal structures (De Lauro et al., 2012). In addition, the seismicity often accompanies the uplift phase of the bradyseism, which involves upward and downward movements of the crust (Orsi et al., 1996). In detail, the LPs events recorded during the 2006 seismic swarm were studied. A careful analysis of the polarization parameters shows that the wavefield becomes perfectly polarized in the direction of the Solfatara crater as the event approaches. The occurrence of LPs is guided by a Possonian process, as is the case for Stromboli volcano, but on different time scales (De Lauro et al., 2012). A further contribution to the modeling is given by the estimate of the energy release, which suggests the existence of privileged scales by the system. Finally, a correlation was demonstrated between the solid solar energy and the energy released by the LPs, indicating that the entire mechanism is modulated on a tidal scale (De Lauro et al., 2012). De Lauro et al. (2013) observed that the time evolution of coarse-grained variables, such as polarization parameters and RMS series, show diurnal tidal modulations. In the RMS analysis, the tides have a mixed character but with a strong dominance of the solar diurnal S1, which may be related to thermal effects (lunar components are negligible). Instead, the azimuth time evolution is characterized by the dominance of solar semidiurnal and solar/lunar diurnal periodicities. This can be an indication that the lunar constituents are a marker of the 2006 miniuplift crisis.

The deformative state of the Campi Flegrei caldera was also evaluated by analysing the tiltmeter signals on long time scales. The background status was indeed evaluated as the response of the caldera plain to tidal stresses on a diurnal, fortnightly and lunar monthly scale. The results show a clear indication that for modelling long-range deformation trend; scientific research should take into account the large-scale oscillation plans, which oscillate under the action of gravitational attraction forces, to be taken into account for a correct evaluation of long-range deformation trends. The specifics of the tidal constituents involved in the dynamics have been recently investigated in De Lauro et al. (2018). They evidenced three tidal constituents by ICA (Bottiglieri et al., 2007, 2010): lunar monthly $\operatorname{Mm}(T=27.55 \mathrm{~d})$; lunar fortnightly Mf $(T=13.66 \mathrm{~d})$; solar diurnal S1 $(T=24 \mathrm{~h})$. The interaction between tidal constituents and ground tilting was highlighted by performing a tilt vector polarization analysis, with the intent to recover information about the preferred directions of oscillation on those long time scales. The estimate of the azimuth of the tilt vector indicated that the ground tilt occurs on preferential planes with an oscillatory pattern, which depends on the periodicity of the corresponding tidal constituent.

Combining the previous results, we derive the spatial distribution of the tilt oscillations identifying the predominant directions along which the ground tilts with diurnal, fortnightly and monthly periodicity. The average results are contained in Fig. 5: this plot indicates how the caldera vibrates, how it oscillates in response to long-period tidal forcing and in which direction.

In light of the previous results, we claim that the earth tides induce ground oscillations with the same periodicity of the tidal constituents. These oscillations are "captured" by the borehole tiltmeters as a ground tilting along well defined planes with nearly a constant orientation. We evidence that the directions of the tilting planes are constrained by the local stress field and are compatible with the distribution and orientation of faults and fractures. The rheology plays an important role in modulating the amplitude of the tidal constituents (De Lauro et al., 2018). Going further, tilt anomalies on medium/long time scales are related to the occurrence rate and energy of VT earthquakes, as well as to the hydrologi- 


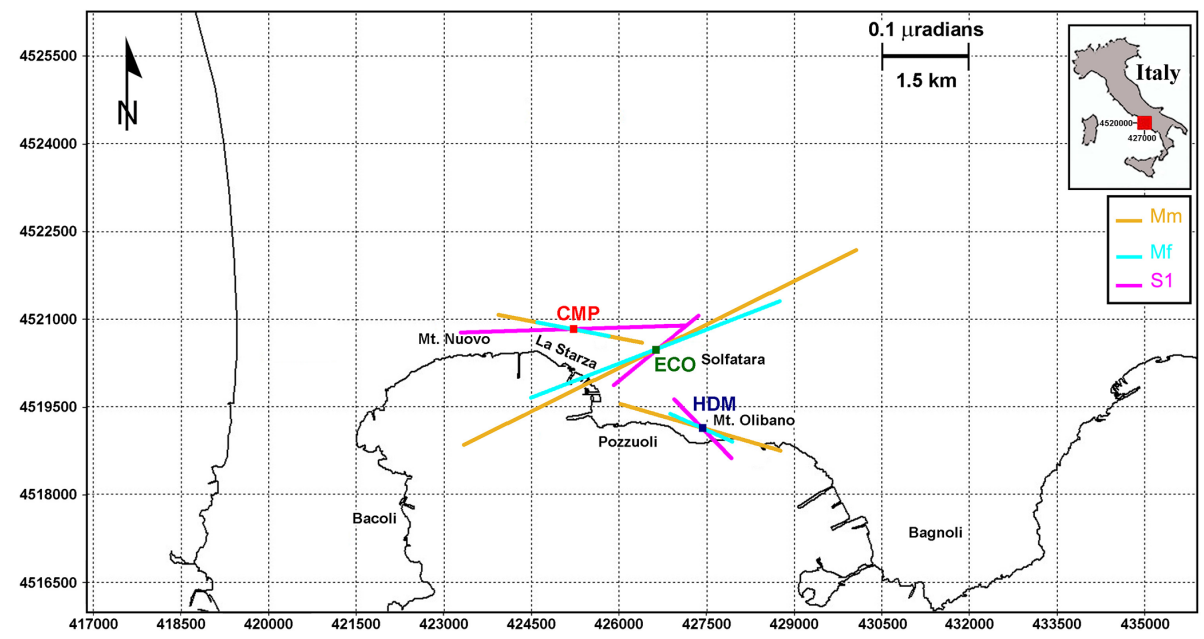

Figure 5. Map of the Campi Flegrei caldera, superimposed on a grid representing the two-dimensional plane of the ground inclinations. For each station, the predominant directions along which the ground tilts with monthly (ochre), fortnightly (cyan) and diurnal (magenta) period are represented (after De Lauro et al., 2018).

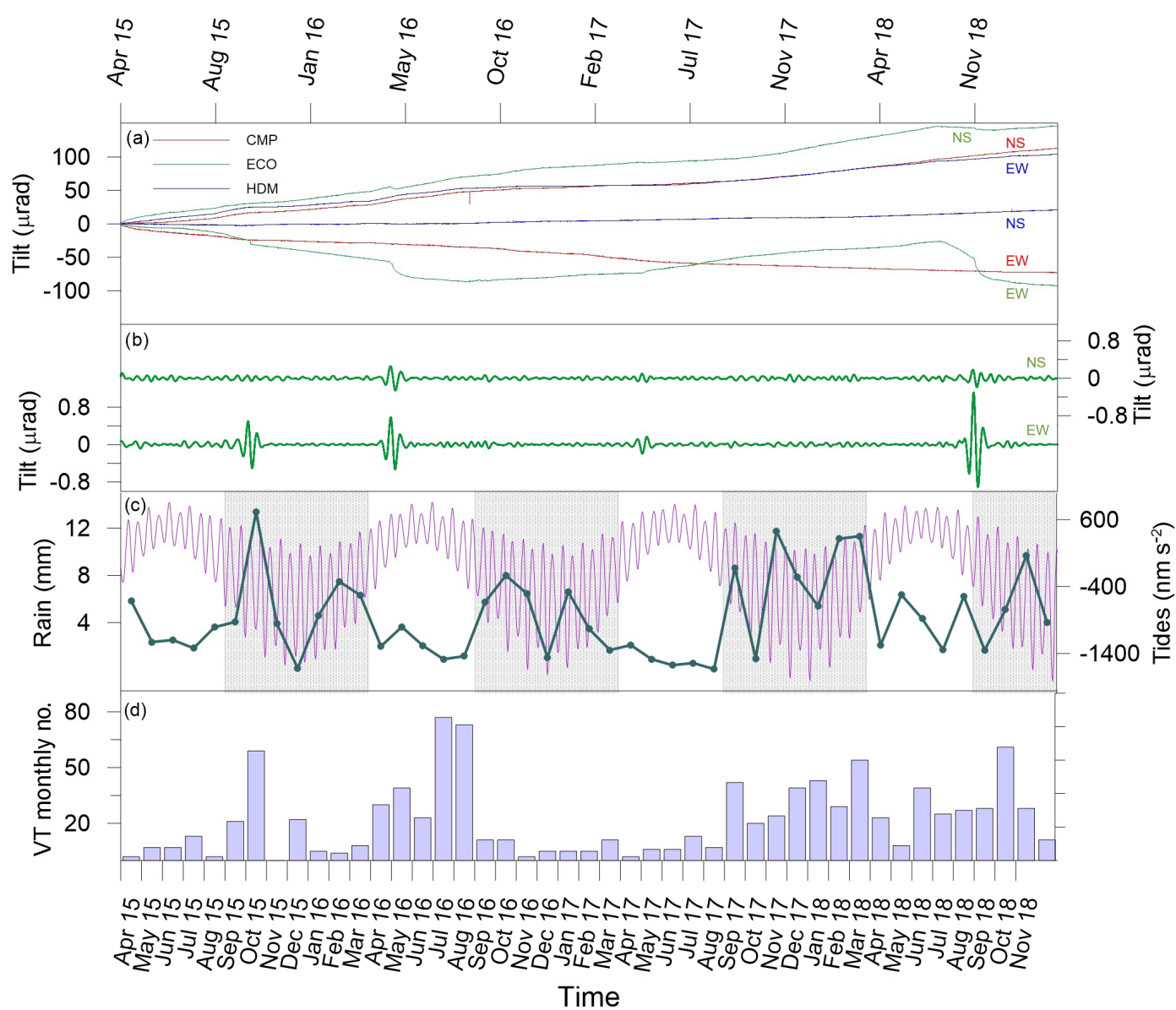

Figure 6. (a) Tilt time series from 1 April 2015 to 31 December 2018; (b) ECO tilt time series filtered in the period band corresponding to the Mf constituent; (c) solid Earth tides (magenta line) and rainfall time series (dark green line). The light-grey boxes in the background mark the wet season of the hydrological year; (d) monthly number of VTs (redrawn after Ricco et al., 2019). 


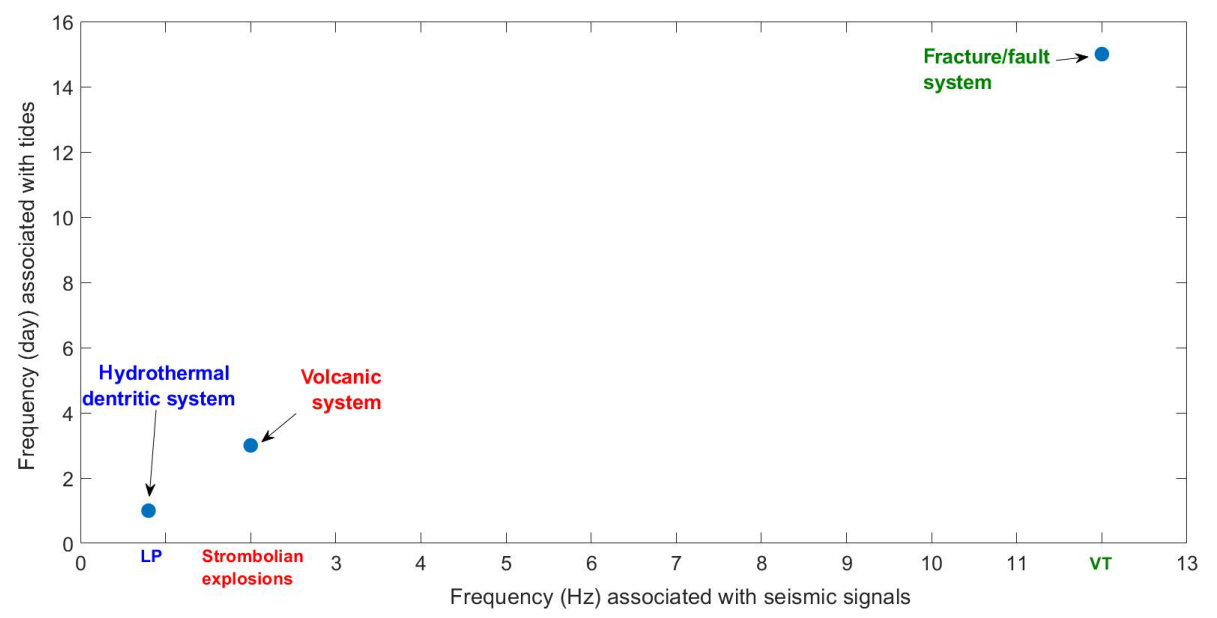

Figure 7. Relation between the tidal periods and the typical frequencies related to the kind of events on that scale and involved system.

cal cycles (Petrosino et al., 2018, 2019; Ricco et al., 2019). These recent studies show that the VT distribution is linked to the rainfall on seasonal scale as it is clearly represented in Fig. 6.

\section{Discussion and conclusions}

Previous analyses evidence the relevance to study tidal oscillations that act in modulating the ground deformation at Campi Flegrei and as a precursor of non-stationary phase at Stromboli. Moreover, the interaction between tides and local deformation, detected by tilt variations, VT and LP occurrence, and/or changes in the explosion-quake statistics, occurs on a variety of scales from diurnal to medium/long period band passing through a $3 \mathrm{~d}$ constituent.

Indeed, at Campi Flegrei caldera, the VT and LP activity are modulated by a tidal contribution on the diurnal and monthly time scales. The oscillations of hydrothermal fluids in the local dendritic system generate the seismic signal at about $1 \mathrm{~Hz}$ in the form of LP and hydrothermal tremor. These signals are modulated by diurnal tidal constituents. In other words, the system should reach a threshold to "become sensitive" to diurnal tides; this background state is determined by the amount of circulating fluids in the hydrothermal system. Indeed, as observed in many volcanic areas, the study of the relation among the hydrothermal activity, seismic and tidal signals is crucial to a better understanding of the fluid-rock interplay that drives the volcano dynamics (Custodio et al., 2003; De Lauro et al., 2018; Cusano et al., 2020).

During VT activity, there can be a joint contribution of tides and rainfall (Petrosino et al., 2018; Ricco et al., 2019). The infiltrating meteoric water can fill pre-existing fractures and/or faults, modifying the fluid pore pressure of the medium and the local stress field. This phenomenon involves tides on the scale of $15 / 30 \mathrm{~d}$ (fortnightly/monthly tides) all over the hydrological year. For this reason we observe a strong seasonality in the VT distribution.

This phenomenon requires to be further investigated, for example estimating the time delays between the infiltration of meteoric water and the ground response, obtaining the transfer function of the medium on tidal scales.

The Strombolian volcanic system shows a phenomenon that evolves on an intermediate time scale case compared with CF. When the 2007 swam occurred, one observed explosion quakes at $2 \mathrm{~Hz}$ linked to the vibration of a smaller part of the conduit due the ascent of the magma within the conduit. The increase of the magmatic fluids which refill a larger part of the conduit is preceded by a $3 \mathrm{~d}$ tide longer than the CF LP case. That is to say, at Stromboli volcano, the tides represent a precursor of a paroxysmal phase.

To summarize, each of these three cases of phenomena synchronizes over tidal scale of different lengths, from seconds to year, as shown in the sketch of Fig. 7.

A better understanding of the tidal effects on the tiltmeter/seismic recordings can help in:

1. improving the outline of the local geology;

2. focusing on the internal sources after the removal of the external tidal effects, thus allowing a better comprehension of the volcanic dynamics;

3. modelling the coupling between ground deformation and earth tides;

4. identification of eventual precursors.

In conclusion, in the studies of the volcanic systems, the interactions among different phenomena (ground deformation, seismic activity, rainfall, earth tides, etc.) over different time scales, considering that the interaction may involve the synchronization of short and long terms, cannot be neglected. In this perspective, further efforts should be done to improve 
multidisciplinary observations by several kinds of geophysical instruments.

Data availability. Data are available on request from the authors.

Author contributions. MF, EDL, SP, SDM conceived the original idea of the present research and developed the conceptual model. MF, EDL, SP elaborated and validated the data. All the authors contributed in writing the manuscript.

Competing interests. The authors declare that they have no conflict of interest.

Special issue statement. This article is part of the special issue "Understanding volcanic processes through geophysical and volcanological data investigations: some case studies from Italian sites".

Review statement. This paper was edited by Paola Cusano and reviewed by Vincenzo Carbone and one anonymous referee.

\section{References}

Aquino, I., Ricco, C., Del Gaudio, C., Augusti, V., and Scarpato, G.: Potenziamento delle reti tiltmetriche nell' area vulcanica campana: Rapporto sull'attività svolta nell' ambito del progetto Vulcamed, Rapporti Tecnici INGV numero 348, ISSN 2039-7941, 2016.

Bottiglieri, M., De Martino, S., Falanga, M., Godano, C., and Palo, M.: Statistics of inter-time of Strombolian explosion-quakes, Europhys. Lett., 72, 492-498, https://doi.org/10.1209/epl/i200510258-0, 2005.

Bottiglieri, M., Falanga, M., Tammaro, U., Obrizzo, F., De Martino, P., Godano, C., and Pingue, F.: Independent component analysis as a tool for ground deformation analysis, Geophys. J. Int.1, 168, 1305-1310, 2007.

Bottiglieri, M., Falanga, M., Tammaro, U., De Martino, P., Obrizzo, F., Godano, C., and Pingue, F.: Characterization of GPS time series at the Neapolitan volcanic area by statistical analysis, J. Geophys. Res., 115, B10416, https://doi.org/10.1111/j.1365246X.2006.03264.x, 2010.

Calvari, S., Spampinato, L., Lodato, L., Harris, A. J., Patrick, M. R., Dehn, J., Burton, M. R., and Andronico, D.: Chronology and complex volcanic processes during the 2002-2003 flank eruption at Stromboli volcano (Italy) reconstructed from direct observations and surveys with a handheld thermal camera, J. Geophys. Res., 110, B02201, https://doi.org/10.1029/2004JB003129, 2005.

Capuano, P., De Lauro, E., De Martino, S., Falanga, M., and Petrosino, S.: Convolutive independent component analysis for processing massive datasets: a case study at Campi Flegrei (Italy),
Nat. Hazards, 86, 417-429, https://doi.org/10.1007/s11069-0162545-0, 2017.

Cesca, S., Braun, T., Tessmer, E., and Dahm, T.: Modelling of the April 5, 2003, Stromboli (Italy) paroxysmal eruption from the inversion of broadband seismic data, Earth Planet. Sc. Lett., 261, 164-178, 2007.

Chiodini, G., Paonita, A., Aiuppa, A., Costa, A., Caliro, S., De Martino, P., Acocella V., and Vandemeulebrouck, J.: Magmas near the critical degassing pressure drive volcanic unrest towards a critical state, Nat. Comm., 7, 13712, https://doi.org/10.1038/ncomms13712, 2016.

Cusano, P., Petrosino, S., and Saccorotti, G.: Hydrothermal origin for sustained long-period (LP) activity at Campi Flegrei Volcanic Complex, Italy, J. Volcanol. Geoth. Res., 177, 1035-1044, 2008.

Cusano, P., Petrosino, S., De Lauro, E., and Falanga, M.: The whisper of the hydrothermal seismic noise at Ischia Island, J. Volcanol. Geoth. Res., 389, https://doi.org/10.1016/j.jvolgeores.2019.106693, 2020.

Custodio, S. I., Fonseca, J. F., d'Oreye, N. F., Faria, B. V., and Bandomo, Z.: Tidal modulation of seismic noise and volcanic tremor, Geophys. Res. Lett., 30, 1816, https://doi.org/10.1029/2003GL016991, 2003.

De Lauro, E., De Martino, S., Del Pezzo, E., Falanga, M., Palo, M., Scarpa, R.: Model for high frequency Strombolian tremor inferred by wavefield decomposition and reconstruction of asymptotic dynamics, J. Geophys. Res., 113, B02302, https://doi.org/10.1029/2006JB004838, 2008.

De Lauro, E., Falanga, M., and Petrosino, S.: Study on the longperiod source mechanism at Campi Flegrei (Italy) by a multiparametric analysis, Phys. Earth Planet. Int., 206-207, 16-30, 2012.

De Lauro, E., De Martino, S., Falanga, M., and Petrosino, S.: Synchronization be-tween tides and sustained oscillations of the hydrothermal system of Campi Flegrei (Italy), Geochem. Geophy. Geosy., 14, 2628-2637, 2013.

De Lauro, E., Petrosino, S., Ricco, C., Aquino, I., and Falanga, M.: Medium and long period ground oscillatory pattern inferred by borehole tiltmetric data: new perspectives for the Campi Flegrei caldera crustal dynamics, Earth Planet. Sc. Lett., 504, 21-29, 2018.

Del Gaudio, C., Aquino, I., Ricciardi, G. P., Ricco, C., and Scandone, R.: Unrest episodes at Campi Flegrei: A reconstruction of vertical ground movements during 1905-2009, J. Volcanol. Geoth. Res., 195, 48-56, 2010.

De Martino, S., Palo, M., and Cimini, G. B.: A statistical study of the Stromboli volcano explosion quakes before and during 2002-2003 eruptive crisis, J. Geophys. Res., 116, B04303, https://doi.org/10.1029/2010JB008047, 2011a.

De Martino, S., Falanga, M., Palo, M., Montalto, M. and Patanè, D.: Statistical analysis of the seismicity during the Strombolian crisis of 2007, Italy: evidence of a precursor in tidal range, J. Geophys. Res., 116, B09312, https://doi.org/10.1029/2010JB007503, 2011 b.

De Martino, S., Errico, A., Palo, M., and Cimini, G. B.: Explosion swarms at Stromboli volcano: A proxy for nonequilibrium conditions in the shallow plumbing system, Geochem. Geophy. Geosy., 13, Q03012, https://doi.org/10.1029/2011GC003949, 2012. 
Falanga, M. and Petrosino, S.: Inferences on the source of longperiod seismicity at Campi Flegrei from polarization analysis and reconstruction of the asymptotic dynamics, Bull. Volcanol., 74, 1537-1551, 2012.

Francalanci, L., Tommasini, S., and Conticelli, S.: The volcanic activity of Stromboli in the 1906-1998 AD period: mineralogical, geochemical and isotope data relevant to the understanding of the plumbing system, J. Volcanol. Geoth. Res., 131, 179-211, 2004.

Gambino, S. and Scaltrito, A.: Volcanic-tectonic seismicity at Stromboli (2005-2016), J. Volcanol. Geoth. Res., 350, 1-6, 2018.

Orsi, G., De Vita, S., and Di Vito, M.: The restless, resurgent Campi Flegrei nested caldera (Italy): constraints on its evolution and configuration, J. Volcanol. Geoth. Res., 74, 179-214, 1996.

Petrosino, S., Cusano, P., and Madonia, P.: Tidal and hydrological periodicities of seismicity reveal new risk scenarios at Campi Flegrei caldera, Sci. Rep., 8, 13808, https://doi.org/10.1038/s41598-018-31760-4, 2018.
Petrosino, S., Ricco, C., De Lauro, E., Aquino, I., and Falanga, M.: Time evolution of medium and long-period ground tilting at Campi Flegrei caldera, Adv. Geosci., accepted, 2019.

Ricco, C., Aquino, I., and Del Gaudio, C.: Ground tilt monitoring at Phlegraean Fields (Italy): A methodological approach, Ann. Geophys., 46, 1297-1314, 2003.

Ricco, C., Petrosino, S., Aquino, I., Del Gaudio, C., and Falanga, M.: Some investigations on a possible relationship between ground deformation and seismic activity at Campi Flegrei and Ischia volcanic areas (Southern Italy), Geosciences, 9, 222, https://doi.org/10.3390/geosciences9050222, 2019.

Saccorotti, G., Petrosino, S., Bianco, F., Castellano, M., Galluzzo, D., La Rocca, M., Del Pezzo, E., Zaccarelli, L., and Cusano, P.: Seismicity associated with the 2004-2006 renewed ground uplift at campi flegrei caldera, Italy, Phys. Earth Planet. Int., 165, 14 24, 2007. 